\title{
Sleep quality and chronic pain in community dwelling older adults: preliminary results
}

\author{
Celuane Oliveira Silva ${ }^{1}$, Glaudson Sá Brandão ${ }^{2}$, Maria Eduarda Moreira Lino ${ }^{3}$, Adriano L. Fonseca ${ }^{4}$, Marcos Mota Silva ${ }^{4}$, \\ Iransé Oliveira-Silva ${ }^{4}$, Francisco K. R. Campos ${ }^{5}$, Anderson S. Silva ${ }^{5}$, Rubens A. Silva ${ }^{6,7}$, Luis V. F. Oliveira ${ }^{4,5}$, Glauber Sá \\ Brandão*1.
}

${ }^{1}$ University of the State of Bahia - UNEB, Department of Education (DEDC-VII), Senhor do Bonfim (BA), Brazil; ${ }^{2}$ IMAIS - Diagnostic and Specialty Clinic, Senhor do Bonfim (BA), Brasil; ${ }^{3}$ Physiotherapy Course, University Center of Anápolis - UniEVANGELICA, Anápolis (GO), Brazil; ${ }^{4}$ Master Degree and PhD Post Graduate Program in Human Movement and Rehabilitation, University Center of Anápolis - UniEVANGELICA, Anápolis (GO), Brazil; ${ }^{5}$ Master Degree and PhD Post Graduate Program in Health Sciences, Santa Casa de São Paulo (SP), Brazil; ${ }^{6}$ Département des Sciences de la Santé, Programme de physiothérapie de l'université McGill offert en extension à l'Université du Québec à Chicoutimi (UQAC), Saguenay, Québec, Canada; ${ }^{7}$ Doctoral and Master's Programs in Rehabilitation Sciences UEL/UNOPAR, LAFUP/UNOPAR, Londrina-PR, Brazil;

\section{ABSTRACT}

Background: The natural process of human aging causes biopsychosocial alterations, which can trigger chronic pain and poor sleep quality in older adults. Considering the high prevalence and possible association between these two clinical conditions, special attention from public health policies is necessary to provide quality aging. Objective: To verify if there is an association between chronic pain and sleep quality among older adults in the community. Methods: A quantitative, cross-sectional study on the association of chronic pain with the sleep quality among older people in the community. Participants were evaluated using the Pittsburgh Sleep Quality Index (PSQI); Visual Analog Pain scale; questionnaires of sociodemographic and clinical data, assessment of cognitive impairment through the Mini Mental State Examination, and anthropometric assessments. The data were submitted to descriptive statistics. The means between the groups of older people with and without chronic pain were compared using the Student's $t$ test for independent samples and Pearson's correlation coefficient ( $r$ ) was used to analyze the association of PSQI with pain intensity. Results: In total, 51 older women were included, with a mean age of $70 \pm 8$ years. The majority had a low level of education (52.9\%), low financial income (64.7\%), and chronic pain (56.9\%). It was found that the older adults with chronic pain presented worse sleep quality when compared those without chronic pain and a moderate $(r=0.595)$ and significant $(p<0.01)$ correlation between sleep quality and the intensity of chronic pain was observed. Conclusion: Older adults in the community with chronic pain present worse sleep quality when compared to the older adults without pain. There is a strong correlation between the intensity of chronic pain and sleep quality in older adults; the greater the intensity of pain, the worse the sleep quality. Trial Registration: Registro Brasileiro de Ensaios Clínicos (REBEC) Identifier: RBR-3cqzfy

Keywords: Older adults, Sleep, Chronic Pain.

\section{BACKGROUND}

Population aging is increasing gradually around the world, due to constant reductions in fertility rates, associated with declining mortality rates. These aspects have changed the age group of the population, contributing to the increase in life expectancy. Projections reveal that by the end of 2020, Brazil will be the sixth country in the world in terms of number of older people and, as aging is a dynamic, progressive process resulting in morphofunctional, biochemical, and psychological alterations, special attention should be paid to health policies to enable healthy aging ${ }^{(1,2,3)}$.

Poor sleep quality and chronic pain are biopsychosocial alterations that are associated with the human aging process. Sleep is fundamental for human beings and changes in its functioning can interfere in physical and emotional aspects, resulting in low cognition, depression, and an increased risk of falls, negatively affecting the living and health conditions of older adults ${ }^{(2)}$.

The percentage of older adults with chronic pain varies from 29.3 to $51.4 \%$. Active pain raises the state of cortical alertness, which can alter the architecture of sleep, in addition to causing discomfort, discouragement, functional dependence, and interference in the activities of daily life $^{(4)}$. The association between poor sleep quality and chronic pain may be relevant to functional alterations in the central nervous system, especially in the thalamus, as it is related to both painful sensations and control of the sleepwake cycle ${ }^{(5)}$. Chronic pain, which leads to high rates of demand for services and health care, progresses with increasing age, becoming one of the major challenges and disorders of public health ${ }^{(4)}$. The older the age and number of chronic diseases, the more likely it is that sleep quality will be compromised ${ }^{(6)}$.

Considering the biological plausibility of the possible relationship between pain and sleep, it becomes relevant to verify if there is an association between chronic pain and sleep quality in older adults in the community, aiming at preventing and promoting the health of this population, in addition to generating information that could subsidize health services in care planning for older adults. 


\section{METHODS}

This study used a methodological focus with a quantitative approach, in a cross-sectional study on the sleep quality of older people in the community with chronic pain. The study was developed based on data from a larger study that analyzed the impact of a home physical exercise program on the sleep quality of older people in the community, which was developed by researchers linked to the group of studies and research in quality of life and healthy aging (QUALES) at the State University of Bahia (BA), Brazil.

Data collection was performed from July to December 2015, after approval from the Ethics Committee in research involving human beings from the Bahia School of Medicine and Public Health - EBMSP, CAAE: 39072514.6.0000.5544. All study participants agreed to participate and signed a free and informed consent form. The research subjects were recruited through radio, social media, and neighborhood associations. The study sample was composed of older people from the community. Inclusion criteria were both sexes, aged equal to or over 60 years, and presenting a sleep disorder. Participants with cognitive impairment according to the Mini-Mental State Examination were excluded $(\mathrm{MMSE})^{(7)}$. Data related to sociodemographic, anthropometric, and self-reported morbidities were collected through individual interviews. The Mini Mental State Examination $(\mathrm{MMSE})^{(7)}$ cognitive impairment instrument, Visual Analog Scale (VAS) ${ }^{(8)}$ for pain intensity, and Pittsburgh sleep quality index (PSQI) ${ }^{(9)}$ were used to assess the older participants.

Regarding anthropometric variables, weight was verified using a Welmy ${ }^{\oplus}$ anthropometric scale with a capacity of 150 kilograms $(\mathrm{Kg})$ placed on a flat surface. The older adults were instructed to wear light clothing and climb barefoot, with empty pockets, onto the center of the base of the scale, with the body erect and weight evenly distributed between the two feet, arms at their sides, and looking forward. Height, in meters $(\mathrm{cm})$, was measured with a vertical stadiometer attached to the scale. The participants placed their backs against the device, legs and feet parallel, arms alongside the body, and palms facing the body. The body mass index (BMI) was determined by the ratio of body mass in kilograms divided by the square of height in meters.

The instrument used to evaluate sleep quality was the Pittsburgh Sleep Quality Index (PSQI) validated for Brazil(9). The PSQI provides a standardized measure of sleep quality, which classifies participants into good or bad sleepers. The instrument consists of questions related to sleep according to the following components: 1 ) subjective sleep quality; 2) sleep latency; 3) sleep duration; 4) usual sleep efficiency; 5) sleep disorders; 6) use of sleeping drugs; 7) daytime sleepiness and dysfunction during the day.
Each component has specific scores and the scores of the seven components are added together to give an overall score ranging from 0 to 21, with scores from 0-4 indicating good sleep quality, 5-10 indicating poor sleep quality, and above 10 indicating a sleep disorder ${ }^{(9)}$. Cognitive impairment was evaluated using the MMSE instrument, which consists of questions that correlate in five dimensions: concentration, language/praxis, orientation, memory, and attention, with a maximum score of 30 points. The cut-off points adopted were 20 points for illiterate older adults, 25 points for those with one to four years of study, 26.5 points for older people with five to eight years of study, 28 points for those with nine to eleven years of study, and 29 points for those with more than eleven years of study ${ }^{(7)}$.

The data were tested for normality through the analysis of the histogram, mean and median, standard deviation, asymmetry and kurtosis and, for confirmation, the Kolmogorov-Smirnov normality test was used. The data were then subjected to descriptive analysis using absolute frequencies and percentages for categorical variables and measures of central tendency and dispersion for numerical variables. The Student's t test for independent samples was used to compare the means between the older adults with and without chronic pain.

Pearson's correlation coefficient ( $r$ ) was used to analyze the association of the global PSQI score with the levels of chronic pain intensity and presented using a box diagram (Box Plot). For decision criteria, a significance level of $5 \%$ ( $p$ $<0.05)$ was adopted.

\section{RESULTS}

Initially, 191 potential participants were recruited from the community, however, 28 refused to participate in the study and 32 did not meet the eligibility criteria, resulting in 51 female participants who were evaluated. A summary of the flow of participants over the course of the study is shown in Figure 1.

The sample was composed exclusively of women, with a mean age of $70 \pm 8$ with low education $(52.9 \%$ with elementary education and $15.7 \%$ illiterate), low income per capita (64.7\% with up to 2 minimum wages) and, in relation to clinical characteristics, it was found that the majority are over the weight considered normal (51\% overweight and $17.6 \%$ with obesity) and $56.9 \%$ present chronic pain. (Table 1). Table 2 presents the comparisons between the older adults with chronic pain and without chronic pain in relation to the components of the PSQI. It can be observed in this comparison that, with the exception of the item referring to the use of sleeping drugs, the older adults with chronic pain presented worse sleep quality when compared to the older adults without chronic pain, with $p<0.001$ for all other components. 


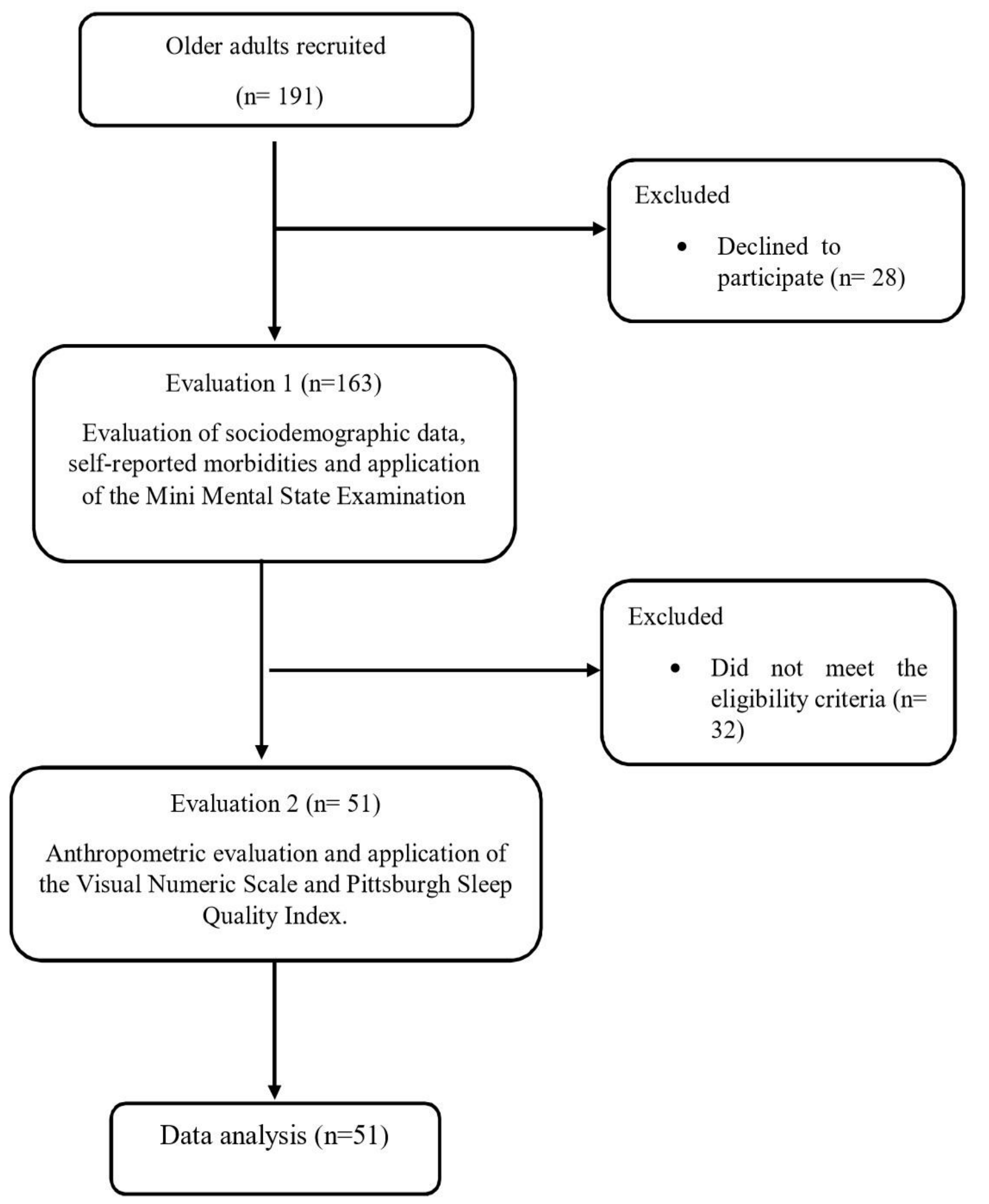

Figure 1. Study flowchart 
Table 1. Sociodemographic characteristics of the studied population

\begin{tabular}{lcc}
\hline Characteristics & $\mathbf{n}(\%)$ & mean $\pm \mathbf{s d}$ \\
\hline Age (years) & & $70 \pm 8$ \\
Schooling & $8(15.7)$ & \\
Illiterate & $27(52.9)$ & \\
Elementary & $13(25.5)$ \\
Secondary & $3(5.9)$ \\
Higher education & \\
Per capita monthly income (MW) & $8(15.7)$ \\
$<1$ & $25(49)$ \\
1 to 2 & $11(21.6)$ \\
$>2$ to 3 & $7(13.7)$ \\
$>3$ & \\
BMl & $16(31.4)$ \\
Normal (18.5 to 24.9) & $26(51)$ \\
Overweight (25.0 to 29.9) & $9(17.6)$ \\
Obesity ( 30$)$ & \\
Chronic pain & $29(56.9)$ \\
Yes & $22(43.1)$ \\
No & \\
\hline
\end{tabular}

Note: ${ }^{*} \mathrm{MW}=$ minimum wages; $\mathrm{BMI}=$ body mass index;

Table 2. Components of the Pittsburgh Sleep Quality Index

\begin{tabular}{lccc}
\hline Components & Without pain & Chronic pain & p \\
\hline Subjective sleep quality & $1.2 \pm 1.0$ & $2.0 \pm 0.7$ & $<0.01$ \\
Sleep latency & $1.2 \pm 0.9$ & $2.2 \pm 0.8$ & $<0.01$ \\
Sleep duration & $1.7 \pm 0.9$ & $2.3 \pm 0.6$ & $<0.01$ \\
Usual sleep efficiency & $1.7 \pm 0.7$ & $2.2 \pm 0.8$ & 0.04 \\
Sleep disorders & $1.8 \pm 0.8$ & $2.4 \pm 0.6$ & 0.01 \\
Use of sleeping drugs & $0.3 \pm 0.1$ & $0.5 \pm 0.3$ & 0.08 \\
Dysfunction during the & $1.1 \pm 0.7$ & $1.6 \pm 0.6$ & 0.01 \\
day & & & $<0.001$ \\
Global score & $9.3 \pm 3.0$ & $12.8 \pm 2.2$ &
\end{tabular}

Note: ${ }^{*}$ PSQI= Pittsburgh Sleep Quality Index 


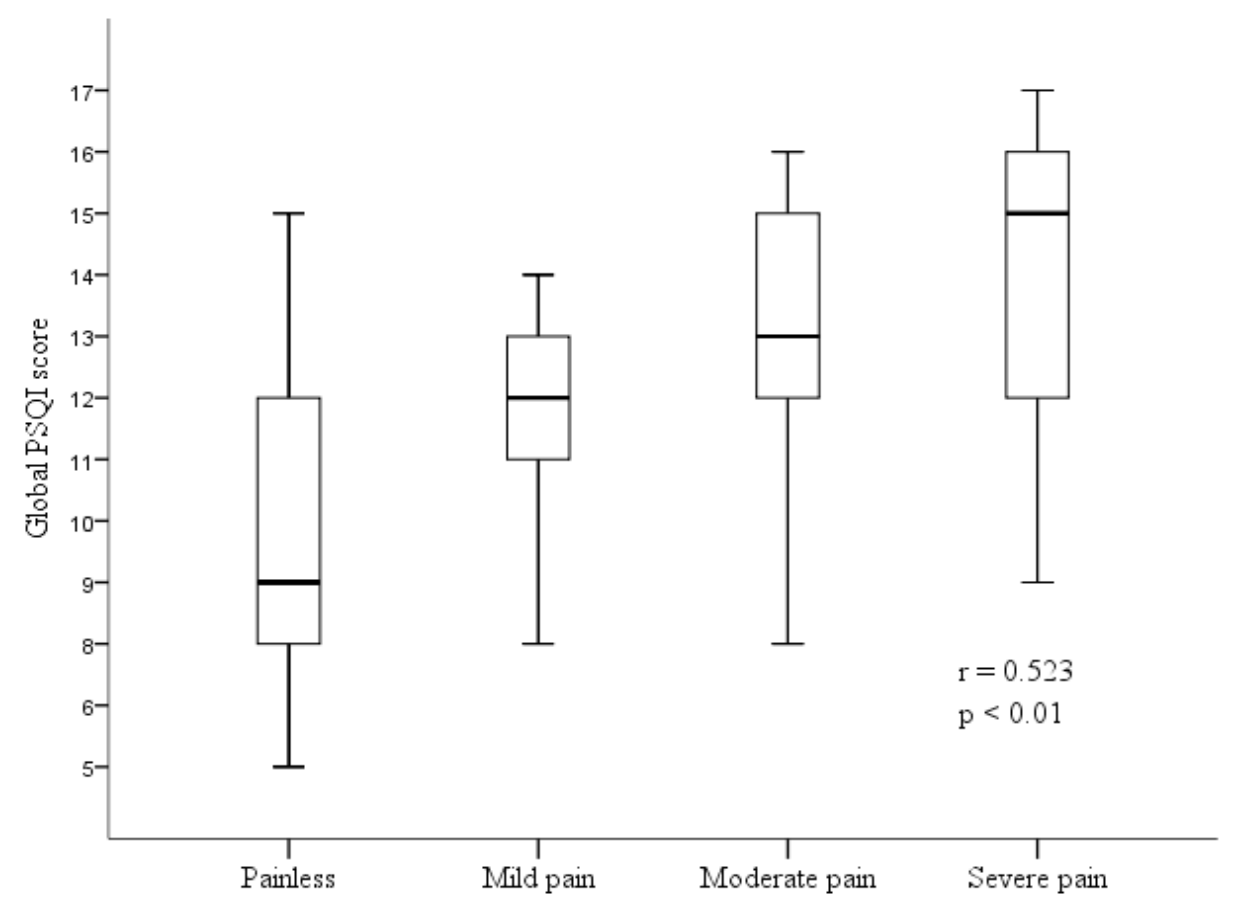

Figure 2. Demonstrates the distribution of the correlation between sleep quality using the global PSQI score and pain intensity using the visual analog scale, showing a moderate $(r=0.523)$ and significant $(p<0.01)$ positive correlation between sleep quality and the intensity of chronic pain. That is, an increase in pain intensity is associated with an increase in the PSQI score, which represents a decline in sleep quality.

\section{DISCUSSION}

The current study demonstrated that older women with chronic pain present worse sleep quality when compared to older women without chronic pain and that there is a positive, moderate, and significant correlation between pain intensity and worsening sleep quality; the greater the pain intensity, the worse the quality of sleep. A cross-sectional study carried out in Sweden found an association of pain with insomnia and that this association is bidirectional. It has also been demonstrated that older people with pain present clinical insomnia and, consequently, a greater number of comorbidities and increased pain, generating high costs in relation to health care when compared to those with less frequency and intensity of pain ${ }^{(10)}$.

The sample of the present study is composed exclusively of older women, with a predominance of the age group from 60 to 69 years and a mean age of $70 \pm 8$ years. This feminization of old age ${ }^{(2)}$ is justified by the fact that women have a lower mortality rate due to external causes, and a low prevalence of smoking and alcohol consumption compared to men, as well as being greater practitioners of self-care ${ }^{(4)}$.

The traditional sleep pattern changes according to age, due to the social and biological alterations of aging itself. There is a transformation in the family cycle, reduction in the number of friends, physical inactivity, daytime sleepiness, and decreased nighttime sleep ${ }^{(11)}$. The sleep time of older adults is much shorter when compared to that of young adults, becoming more fragmented in relation to the duration, distribution of stages, and rhythm of sleep ${ }^{(4)}$.

Alterations in circadian rhythm and neurodegenerative processes cause sleep disorders and reduced cognitive function. Thus, older adults without cognitive changes are likely to experience greater increases in sleep duration. Older people with difficulty sleeping are more exposed to depressive symptoms and, consequently, greater risks for cognitive impairment and dementia ${ }^{(5)}$.

Some studies have shown that older people with poor sleep quality are more likely to present generalized chronic pain and that poor sleep increases sensitivity to pain, in addition to demonstrating that there is a strong and significant association between chronic pain and sleep quality $(12,13,14)$, corroborating the findings of the present study. The association of chronic pain with insomnia and daytime sleepiness is related to alterations in the physiological process of human aging(4), which can affect the central nervous system, impairing the cognitive, sensory, and motor 
functions of older adults ${ }^{(12)}$. In agreement with the present study, Felix et al. found that $49 \%$ of the oldest old patients with chronic pain presented poor sleep ${ }^{(14)}$.

In older adults, chronic pain is mainly related to musculoskeletal and osteoarticular diseases. However, in many cases, complaints of pain by older adults are ignored, resulting in long suffering, which can interfere with the quality of sleep and the integrality of biopsychosocial wellbeing ${ }^{(15)}$, as demonstrated in the results of the present study. The MOBILIZE study found that older people with pain in various parts of the body and, consequently, many comorbidities, present worse sleep quality. This makes it possible to specify that chronic pain and poor sleep quality are bidirectional, being able to interfere in the quality of life of older adults ${ }^{(16)}$. Considering the difficulty in treating chronic pain in older adults, due to the complexity of physiological alterations that occur during the aging process, it is essential to understand these changes, as they act as precursors to the onset of pain and poor sleep quality $^{(4)}$. Therefore, it is necessary that health professionals carry out more active interventions in order to prevent, diagnose, and treat pain and sleep quality. Since these are important clinical conditions, their bidirectional association must be understood and respected when carrying out care planning for older adults ${ }^{(17)}$. This study has some limitations due to the use of a cross-sectional design in which only female patients were involved, so the associations between chronic pain and sleep quality among older adults should be interpreted with caution. Although we know that the studied variables have a bidirectional relationship ${ }^{(16)}$, the results do not allow causal inference. Although we used validated questionnaires, another limitation was the use of self-reported measures, increasing the possibility of measurement errors, recall bias, and the effect of social desire ${ }^{(18)}$. Future studies should use objective methods such as polysomnography and longitudinal methodology to better capture and understand the causality between chronic pain and sleep quality in older adults.

\section{CONCLUSION}

Given the above, it is possible to verify the feminization of old age, a predominance of low socioeconomic class and low education, with the majority being overweight and presenting chronic pain. It was identified that older people with chronic pain present worse sleep quality when compared to older adults without pain and that there is a strong correlation between the intensity of chronic pain and sleep quality of older adults; the greater the intensity of pain, the worse the sleep quality.

\section{Acknowledgements}

The authors would like to thank the National Council for Scientific and Technological Development (CNPq), Brazil and the Program for Supporting Teacher and Administrative Technical Training at the State University of Bahia - UNEB, for funding the research. We would also like to thank the older adults who agreed to participate in the study and the members of the research group: "Quality of life and Healthy Aging" (QUALES) for their dedication to data collection.

\section{AUTHORS' CONTRIBUTION:}

GSB*, LVFO, GSB, COS: Contributed to the elaboration of the design, development of the study and data acquisition. COS, GSB*, Contributed to data tabulation. MEML, ALF, MMS, FKRL, ASS, RAS: Contributed to the critical review, correction and approval of the final version; All authors: Contributed to the development of study and data analysis and approval of the final version.

\section{ORCID:}

Celuane Oliveira Silva https://orcid.org/0000-0002-0357-3037 Maria Eduarda M. Lino https://orcid.org/0000-0002-0210-8512 Luís V F Oliveira https://orcid.org/0000-0002-3852-9415 Glauber S Brandão https://orcid.org/0000-0003-4462-0861

FUNDING: This study was funded by the State University of Bahia, through the Support Program for the Training of Teachers and Administrative Technicians (PAC); LVFO received grants for Research Productivity, modality PQID; Process no. 312731/2018-3 from the National Council for Scientific and Technological Development (local acronym $\mathrm{CNPq}$ ), Brazil. ASS and FKRC receive scholarships from CAPES - Coordenação de Aperfeiçoamento de Pessoal de Nível Superior. These grants did not influence any of the stages of the study, including the conception, development, analysis, and interpretation of the results.

CONFLICT OF INTEREST: The authors report no conflict of interest

\section{REFERENCES}

1. Sousa FJD, Andrade FS. Perfil de los adultos mayores usuarios de atención primaria. Revista Cuidarte. 2017;8(2):1573-1581.

2. Berlindes, AK. Envelhecimento populacional, cuidado e cidadania: velhos dilemas e novos desafios. Soc. estado. 2012;27(1):165-180.

3. Olívia GLF, Silvana CM, Sônia MGC, Antonia OS, Maria Adelaide SPM. Envelhecimento ativo e sua relação com a independência. Texto Contexto Enferm. 2012;21(3):513-8.

4. Pereira LV, Vasconcelos PP, Souza LAF, Pereira GA, Nakatani AYK, Bachion MM. Prevalência, intensidade de dor crônica e autopercepção de saúde entre idosos: estudo de base populacional. Rev. Latino-Am. Enfermagem.2014;22(4):662-9.

5. Gisele SML, Philippe M, Marleide MG. Transtorno do sono. Atualização (1/2). Revista Brasileira de Neurologia. 2017;53(3):19-30. 
6. Ferretti F, Santos DT, Giuriatti L, Gauer APM, Teo CRPA. Sleep quality in the elderly with and without chronic pain. Sociedade Brasileira para o Estudo da Dor. 2018;1(2):141-6.

7. Brucki SMD, Nitrin R, Caramelli $P$, Bertolucci PHF, Okamoto IH. Suggestions for utilization of the minimental state examination in Brazil. Arq Neuropsiquiatr. 2003;61(3-B):777-781.

8. Araujo LC, Romero B. Pain: evaluation of the fifth vital sign. A theoretical reflection. Revista Dor 2015;16(4):291-6.

9. Bertolazi AN, Fagondes SC, Hoff LS et al. Validation of the Brazilian Portuguese version of the Pittsburgh sleep quality index. Sleep Med. 2011;12(1):70-5.

10. Malek-Ahmadi M, Kora K, O'Connor K, Schofield S, Coon D, Nieri W. Longer self-reported sleep duration is associated with decreased performance on the montreal cognitive assessment in older adults. Aging Clinical and Experimental Research. 2015;28(2):333337.

11. Olivência SA, Barbosa LGM, Cunha MR, Silva LJ. Tratamento farmacológico da dor crônica não oncológica em idosos: Revisão integrativa. Rev. Bras. Geriatr. Gerontol. 2018;21(3): 383-393.

12. Paladini A, Fusco M, Coaccioli S, Skaper SD, Varrassi G. Chronic pain in the elderly: the case for new therapeutic strategies. Pain Physician. 2015;18(5):86376.

13. Liu XK, Xiao SY, Zhou L, Zhou MHW, Liu H. Sleep quality and covariates as predictors of pain intensity among the general population in rural China. Journal of Pain Research. 2018;11:857-866.

14. Felix RH, Almeida CBL, Cremaschi RC, Coelho FM, Santos FC. Pain-induced depression is related to overestimation of sleep quality in a very elderly population with pain. Arq Neuropsiquiatr 2017;75(1):25-29.

15. Santos AA, Schlosser TCSM, Ceolim MF, Pavarini SCL. Sleep, fragility and cognition: a multicenter study with Brazilian elderly. Rev Bras Enferm. 2013;66(3):351-7.

16. Chen Q, Hayman LL, Shmerling RH, Bean JF, Leveille SG. Characteristics of chronic pain associated with sleep difficulty in older adults: the Maintenance of Balance, Independent Living, Intellect, and Zest in the Elderly (MOBILIZE) Boston study.J Am Geriatr Soc. 2011;59(8):1385-1392.

17. Veras RP. Estratégias para o enfrentamento das doenças crônicas: um modelo em que todos ganham. Rev Bras Geriatr Gerontol. 2011;14(4):779-86.

18. Shephard R.J. Limits to the measurement of habitual physical activity by questionnaires. Br. J. Sports Med. 2003;37:197-206. 